\title{
Nuclear Acoustic Resonance in Metals
}

\author{
V. Müller and U. Bartell \\ Institut für Atom- und Festkörperphysik der Freien Universität Berlin, \\ Berlin, Germany
}

Received August 28, 1978

\begin{abstract}
A comprehensive quantum theoretical treatment of nuclear acoustic resonance (NAR) in metals is presented for the first time. Basic equations describing the NAR-absorption and NAR-dispersion are derived from the sound induced perturbation Hamiltonian $\mathbb{T}(t)$ by applying a generalized form of the "Kubo susceptibility". It is shown that in metals, where a sound wave may induce nuclear magnetic dipole and nuclear electric quadrupole transitions simultaneously, the appearance of interference terms enables one to determine not only the absolute values but also the signs of the gradient-elastic tensor components. Explicit expressions are displayed for the dipolar, quadrupolar and interference contributions to the generalized NAR susceptibility in cubic metals. As an example the derivative of the expected ${ }^{93} \mathrm{Nb}$ NAR-absorption line $(|\Delta m|=1)$ is calculated for different signs of the gradient elastic tensor component $S_{44}$.
\end{abstract}

\section{Introduction}

Since the first observation of nuclear acoustic resonance (NAR) in nonconducting materials by Bolef and Menes [1], and in metals by Gregory and Bömmel [2], a large number of NAR experiments has been performed which show that nuclear acoustic spin transitions may be induced via two relevant spin-phonon coupling mechanisms. One of them is the familiar nuclear electric quadrupole interaction [3-4] with the sound induced dynamic electric field gradient (DEFG) tensor $\overleftrightarrow{V}(\vec{r}, t)$, the other one is the nuclear magnetic dipole interaction with the sound induced $\mathrm{rf}$ magnetic field $\vec{b}(\vec{r}, t)$, originally suggested by Quinn and Ying [5]. This dipolar coupling mechanism, however, first observed by Buttet et al. [6] in aluminum, solely may be observed in the presence of a strong de magnetic field and in good conductors only. Consequently, the relevant nuclear spin-phonon Hamiltonian $\mathbb{h}(t)$ of an ultrasonically excited metal becomes

$\mathrm{Ih}(t)=\mathrm{Ih}_{D}(t)+\mathbb{I h}_{Q}(t)$

with

$\mathrm{Ih}_{D}(t)=-\int d^{3} \vec{r} \mathrm{~m}(\vec{r}) \cdot \vec{b}(\vec{r}, t)$

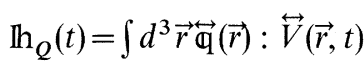

where $\vec{m}(\vec{r})$ is the operator of the nuclear spin magnetization and $\overleftrightarrow{\mathbb{q}}(\vec{r})$ the tensor operator of the nuclear electric quadrupole-moment density.

Although the nuclear spin-phonon Hamiltonian of metals and isolators differs only in the additional magnetic dipole term $\mathrm{Ih}_{D}$, this term gives rise to some fundamental difficulties which may explain the lack of a full theory of NAR in metals. One of these difficulties occurs because the sound induced rf magnetic field is a response quantity and therefore cannot be treated as an external quantity (like in conventional NMR), so that phase effects must be considered when deriving from the perturbation Hamiltonian (1) the correct expressions for the resulting change in the ultrasonic attenuation and sound velocity. Moreover, as a result of the simultaneous action of both the sound induced rf magnetic field and the DEFG, the equations of motion of the nuclear spin magnetization and the nuclear electric quadrupole-moment density are coupled $[4,7]$, thus giving rise to interference terms [8] which essentially may influence the observed NAR lines.

Previous theories of NAR in metals [3-5, 9-17], however, are restricted to either pure dipole- or pure quadrupole-NAR, where relevant interference effects do not occur. With one exception, there is further- 
more a striking discrepancy between the experimental results and the final expressions of previous quantum theoretical treatments of dipole-NAR in metals, since in these theories the above-mentioned phase effects are treated in an incomplete manner. Hence, it is our main purpose to present a comprehensive quantum theoretical treatment of NAR in metals, including both interference and phase effects, and to derive in a straight forward manner explicit expressions for the change in the ultrasonic attenuation and sound velocity from the perturbation Hamiltonian (1). Furthermore, we will show that the investigation of the interference contributions to the $|\Delta m|=1$ NAR lines should be a particular useful tool in determining not only the absolute values, but also the sign of the DEFG tensor components which is of particular interest when studying the conduction electron contributions to the nuclear spin-phonon coupling in metals [18].

\section{Generalized NAR Susceptibility}

Among the quantum theoretical treatments of irreversible processes, dealing with linear response of physical systems which are subjected to an external "driving force" $f(t)$ and have undergone an external excitation of the form

$\mathbb{h}_{f}(t)=\mathbb{W} f(t)$

(where the operator $\mathbb{W}$ is independent of $f(t)$ and constant with respect to the time variable $t$ ), Kubo's concept of generalized susceptibility [19, 20] (represents one of the most powerful tools correlating an external perturbation Hamiltonian $\mathbb{h}_{f}(t)$ with relevant experimental quantities. This concept, however, successfully used as well in NMR [19] as in NAR $[4,21]$, fails if the perturbation Hamiltonian is not of the form (4) for, in that case, it is impossible to give the absorbed power in terms of the "Kubo susceptibility" $\chi_{W}$ which for $\Omega \neq 0$ is defined by

$-\operatorname{Tr}\{\rho(\Omega) \mathbb{W}\}=\chi_{W}(\Omega) f(\Omega)$

where $\rho$ is the density operator and the argument $\Omega$ stands for the time-Fourier transform. On the other hand, when taking into account that in NAR the sound wave is considered to be the external "driving force" $f(t)$ and regarding that due to the electronphonon interaction the sound induced electrostatic and electromagnetic fields in general are phase shifted with respect to $f(t)$, from (2), (3) and (1) it becomes obvious that without a generalization, Kubo's treatment cannot be applied to NAR in metals.
However, when analyzing the basic assumptions of linear response theory, after which the response $\Delta A(t)$ $=\Delta\{\operatorname{Tr}\{\rho \mathbb{A}\}\}$ of an arbitrary (time independent) physical quantity $\mathbb{A}$ of a driven system must be a linear function of the "driving force" $f(t)$, it is easy to see that this premise is equivalent to the condition, of $\rho(t)$ to be a linear response quantity itself. Nevertheless, this condition does not imply a perturbation Hamiltonian of the form (4). In fact, when starting from the master equation

$i \hbar \frac{\partial \rho}{\partial t}=\left[\mathbb{H}_{0}+\mathbb{h}(t), \rho(t)\right]$

and keeping only first order terms in $\mathrm{Th}(t)$, it is easy to show that the time-Fourier transform at $\Omega \neq 0$ comes out to be

$\rho(\Omega)=\frac{1}{i \hbar}\left[\tilde{\mathrm{h}}(\Omega), \rho_{0}\right]$

with*

$\tilde{\mathrm{h}}(\Omega)=\int_{0}^{\infty} d t e^{-i \Omega t} e^{-\frac{i}{\hbar} \mathbb{H}_{0} t} \operatorname{hh}(\Omega) e^{\frac{i}{\hbar} \mathbb{H _ { 0 } t}}$

where $\mathrm{IH}_{0}$ is the Hamiltonian of the unperturbed physical system under regard and $\rho_{0}$ is the statistical operator at $t=-\infty$ which is diagonal in the energy basis. Hence, as a consequence of (7) and (8), the Fourier transform of the most general perturbation Hamiltonian which is compatible with the assumption of linear response of $\rho(t)$ to $f(t)$, must be of the form

$\operatorname{Ih}(\Omega)=\mathbb{W}(\Omega) f(\Omega)$

thus leading with the convolution theorem to

$\operatorname{lh}(t)=\frac{1}{2 \pi} \int_{-\infty}^{\infty} d \tau \mathbb{W}(\tau) f(t-\tau)$

where $\mathbb{W}(\Omega)$ is the Fourier transform of the "response operator" W $(\tau)$ which is independent of $f(\tau)$ and, due to the principle of causality, is zero for $\tau<0$.

Consequently it is straight forward to define a "generalized susceptibility" $\chi_{h}(\Omega)$ by substituting $\mathbb{W}$ in (5) by $\operatorname{lh}(\Omega) / f(\Omega)$, for in that case the resulting expression - just as the "Kubo susceptibility" $\chi_{W}$ - becomes independent of $f(\Omega)$. With (5) the "generalized susceptibility" then gets the form

$\chi_{h}(\Omega)=-\frac{\operatorname{Tr}\{\rho(\Omega) \operatorname{lh}(\Omega)\}}{f(\Omega) f(\Omega)}$

* Note that in the Fourier space $\mathrm{Ih}(\Omega)$ is not a Hermitean but is adjoint to $\mathbb{t h}(-\Omega)$ 
which indeed is a generalization of the Kubo expression since now, the defining equation is not restricted to a perturbation Hamiltonian of the form (4).

Taking into account that in cw acoustic experiments the not symmetrized strain tensor $\overleftrightarrow{\varepsilon}^{\prime}$ only has one nonzero component $\varepsilon^{\prime}$, the concept of generalized susceptibility easily may be applied to NAR when $\varepsilon^{\prime}$ is regarded as the "driving force" $f(t)$. Four our further considerations, however, it is more convenient to dispose of $f^{2}(t)$ such as to be an energy like quantity, for in that case, the resulting generalized susceptibility - just as the conventional nuclear magnetic spin susceptibility - becomes a dimensionless quantity which immediately may be compared with other (dimensionless) susceptibilities. Hence, bearing in mind that the acoustic energy is

$\rho_{s}\left\{c_{a}^{2} \int d^{3} \vec{r}\left[\varepsilon^{\prime}(\vec{r}, t)\right]^{2}+\int d^{3} \vec{r}\left[\vec{v}_{i}(\vec{r}, t)\right]^{2}\right\} / 2$,

and regarding that for an ultrasonic standing wave the identity

$\rho_{s}\left\{c_{a}^{2} \int d^{3} \vec{r}\left[\varepsilon^{\prime}(\vec{r}, \Omega)\right]^{2}+\int d^{3} \vec{r}\left[\vec{v}_{i}(\vec{r}, \Omega)\right]^{2}\right\}=0$

must hold for $\Omega \neq 0$, we either may put

$[f(\Omega)]^{2}=\rho_{s} c_{a}^{2} \int_{\left(V_{s}\right)} d^{3} \vec{r}\left[\varepsilon^{\prime}(\vec{r}, \Omega)\right]^{2}$

or

$[f(\Omega)]^{2}=-\rho_{s} \int_{\left(\boldsymbol{V}_{s}\right)} d^{3} \vec{r}\left[\vec{v}_{i}(\vec{r}, \Omega)\right]^{2}$

where $\rho_{s}$ is the mass density of the specimen, $V_{s}$ the volume of the sample containing the nuclei, $\vec{v}_{i}$ is the velocity of the lattice displacement and $c_{a}$ is the phase velocity of sound. Thus, combining (11) and (12), the dimensionless "generalized NAR susceptibility" either may be defined as

$\chi_{\mathrm{NAR}}(\Omega)=-\frac{\operatorname{Tr}\{\rho(\Omega) \operatorname{hh}(\Omega)\}}{\rho_{s} c_{a}^{2} \int_{\left(V_{s}\right)} d^{3} \vec{r}\left[\varepsilon^{\prime}(\vec{r}, \Omega)\right]^{2}}$

or

$\chi_{\mathrm{NAR}}(\Omega)=\frac{\operatorname{Tr}\{\rho(\Omega) \operatorname{hh}(\Omega)\}}{\rho_{s} \int_{\left(V_{s}\right)} d^{3} \vec{r}\left[\vec{v}_{i}(\vec{r}, \Omega)\right]^{2}}$.

Applying this definition to NAR in metals, where the perturbation Hamiltonian (1) is of the form (10) since $\vec{b}(\vec{r}, t)$ and $\overleftrightarrow{V}(\vec{r}, t)$ are linear response quantities with respect to $\varepsilon^{\prime}(\vec{r}, t)$, by aid of Eqs. (1), (7) and (13) and with the abbreviations

$\chi_{\mathrm{NAR}}^{(D)}(\Omega)=\chi_{\mathrm{NAR}}^{(D D)}=-\frac{1}{i \hbar} \frac{\left\langle\left[\mathrm{T}_{D}(\Omega), \tilde{\mathrm{h}}_{D}(\Omega)\right]\right\rangle_{0}}{\rho_{s} c_{u}^{2} \int_{\left(V_{s}\right)} d^{3} \vec{r}\left[\varepsilon^{\prime}(\vec{r}, \Omega)\right]^{2}}$
$\chi_{\mathrm{NAR}}^{(Q)}(\Omega)=\chi_{\mathrm{NAR}}^{(Q Q)}=-\frac{1}{i \hbar} \frac{\left\langle\left[\mathrm{h}_{Q}(\Omega), \tilde{\mathrm{h}}_{Q}(\Omega)\right]\right\rangle_{0}}{\rho_{s} c_{a}^{2} \int_{\left(V_{s}\right)} d^{3} \vec{r}\left[\varepsilon^{\prime}(\vec{r}, \Omega)\right]^{2}}$

$\chi_{\mathrm{NAR}}^{(\mathrm{Int})}(\Omega)=\chi_{\mathrm{NAR}}^{(Q D)}+\chi_{\mathrm{NAR}}^{(D Q)}$

$=-\frac{1}{i \hbar} \frac{\left\langle\left[\mathrm{h}_{D}(\Omega), \tilde{\mathrm{h}}_{Q}(\Omega)\right]+\left[\mathrm{h}_{Q}(\Omega), \tilde{\mathrm{h}}_{D}(\Omega)\right]\right\rangle_{0}}{\rho_{s} c_{a}^{2} \int_{\left(\boldsymbol{V}_{\boldsymbol{s}}\right)} d^{3} \vec{r}\left[\varepsilon^{\prime}(\vec{r}, \Omega)\right]^{2}}$

we obtain

$\chi_{\mathrm{NAR}}(\Omega)=\chi_{\mathrm{NAR}}^{(D)}(\Omega)+\chi_{\mathrm{NAR}}^{(Q)}(\Omega)+\chi_{\mathrm{NAR}}^{(\mathrm{Int})}(\Omega)$

where the expectation value $\langle\ldots\rangle_{0}=\operatorname{Tr}\left\{\rho_{0} \ldots\right\}$ is to be evaluated in the equilibrium spin ensemble, $\chi_{\mathrm{NAR}}^{(D)}$ is the NAR dipole susceptibility, $\chi_{\text {NAR }}^{(Q)}$ the NAR quadrupole susceptibility and $\chi_{\mathrm{NAR}}^{(\mathrm{Int})}$ is an interference term which takes into account that the equations of motion of the nuclear spin magnetization and of the nuclear electric quadrupole moment density in general are coupled. Since the NAR susceptibility not only contains the traditional terms quadratic with respect to the absolute values of the matrix elements of $\mathbb{h}_{D}$ and $\mathrm{th}_{Q}$, but also the interference term (14c) whose sign depends on both the sign of the gyromagnetic ratio and the sign of the DEFG tensor components, it is worth mentioning that the $|\Delta m|=1$ NAR lines are expected to be different whether the electric field gradient terms are positive or negative.

Finally we give the corresponding expressions for a model of wide practical relevance, where the nuclear spin system consists of $N$ identical particles having $I>\frac{1}{2}$ and static effects of the surrounding matter are assumed to be small perturbations on the Zeeman splitting. For long-wave phonons, then, we have

$$
\begin{aligned}
& \operatorname{Tr}\left\{\rho_{0}[\operatorname{lh}(\Omega), \tilde{\mathrm{h}}(\Omega)]\right\} \\
& =\frac{N}{V_{s}} \int_{\left(V_{s}\right)} d^{3} \vec{r} \int_{-\infty}^{\infty} d a_{D} \int_{-\infty}^{\infty} d a_{Q} g_{D}\left(a_{D}\right) g_{Q}\left(a_{Q}\right) \\
& \cdot \operatorname{Tr}\left\{\hat{\mathbb{P}}_{0}[\hat{\mathrm{h}}(\vec{r}, \Omega), \hat{\tilde{\mathrm{h}}}(\vec{r}, \Omega)]\right\}
\end{aligned}
$$

where $g_{Q}\left(a_{Q}\right)$ and $g_{D}\left(a_{D}\right)$ are the normalized distribution functions of the quadrupolar frequencies $a_{Q}$ and the dipolar frequencies $a_{D}$, $\hat{\mathrm{h}}$ is the one-particle perturbation Hamiltonian $\widehat{\mathbb{h}}(\vec{r}, \Omega)=\widehat{\mathbb{h}}_{D}(\vec{r}, \Omega)+\widehat{\mathrm{h}}_{Q}(\vec{r}, \Omega)$,

$\hat{p}_{0}=\exp \left(-\hat{\mathbb{H}}_{0} / k_{B} T\right) / \operatorname{Tr}\left\{\exp \left(-\hat{\mathbb{H}}_{0} / k_{B} T\right)\right\}$

is the equilibrium operator and

$\widehat{\tilde{\mathrm{h}}}(\vec{r}, \Omega)=\int_{0}^{\infty} d t e^{-i \Omega t} e^{-\left(\frac{i}{h}\right) \hat{\mathrm{H}}_{0} t} \hat{\mathrm{h}}(\vec{r}, \Omega) e^{\left(\frac{i}{h}\right) \hat{\mathrm{H}}_{0} t}$.

Here $\widehat{\mathbb{H}}_{0}=\hat{\mathbb{H}}_{z}+\widehat{\mathbb{I}}_{D}+\hat{\mathbb{H}}_{Q}$, where $\hat{\mathbb{H}}_{z}=-\gamma \hbar B_{0} \mathbb{I}_{z}$ is the Zeeman part, $\gamma$ the gyromagnetic ratio and (in the first order approximation) $\hat{\mathbb{H}}_{Q}=a_{Q} \hbar\left(\mathbb{I}_{z}^{2}-I(I+1) / 3\right)$ is the electric quadrupole part with the quadrupole 
frequency

$a_{Q}=\frac{3 e_{0} Q V_{z z}^{(0)}}{4 I(2 I-1) \hbar}$

$e_{0}$ is the elementary electric charge, $Q$ the nuclear electric quadrupole moment and $V_{z z}^{(0)}$ is the component of the static EFG at the site of a given nucleus in the direction of the external magnetic field $\vec{B}_{0}$. The term $\hat{\mathbb{H}}_{D}$ describes the inhomogeneous broadening via the magnetic dipolar interaction of the nuclear spins and, in a simple model, may be assumed to be [22] $\hat{\mathbb{H}}_{D}=a_{D} \hbar \mathrm{II}_{z}$, where

$a_{D}=-\gamma B_{D z}$

and $B_{D z}$ is the local dipolar field component at the site of the given nucleus in the direction of $\vec{B}_{0}$. Thus, summing up the terms given above, the Hamiltonian of a single spin becomes

$\widehat{\mathrm{H}}_{0}=-\gamma \hbar B_{0} \mathrm{I}_{z}+\hbar a_{D} \mathrm{II}_{z}+\hbar a_{Q}\left(\mathrm{II}_{z}^{2}-I(I+1) / 3\right)$

so that with $\mathrm{II}_{z}|m\rangle=m|m\rangle$, (14), (15), (16) and $\left\langle m\left|\hat{p}_{0}\right| m\right\rangle=N_{m} / N \quad$ (where $N_{m}$ is the occupation number of the eigenstate $|m\rangle)$ the generalized NAR susceptibility as well as the resulting NAR lineshape can be computed by using standard treatments familiar in NMR [23].

From (14) and (16) we then obtain

$$
\begin{aligned}
& \chi_{\mathrm{NAR}}^{(\alpha \beta)}(\Omega)=\frac{\pi}{\hbar \rho_{s} c_{a}^{2} \int_{\left(V_{s}\right)} d^{3} \vec{r}\left[\varepsilon^{\prime}(\vec{r}, \Omega)\right]^{2}} \\
& \cdot \sum_{m, m^{\prime}} \frac{N_{m}^{(0)}-N_{m^{\prime}}^{(0)}}{V_{s}} \int_{\left(V_{s}\right)} d^{3} \vec{r} \\
& \cdot\left\langle m^{\prime}\left|\widehat{\mathrm{h}}_{\alpha}(\vec{r}, \Omega)\right| m\right\rangle\left\langle m\left|\hat{\mathrm{h}}_{\beta}(\vec{r}, \Omega)\right| m^{\prime}\right\rangle g\left(\Omega-\omega_{m^{\prime} m}\right)
\end{aligned}
$$

where $\alpha, \beta$ stand for the indices $D, Q$,

$$
\begin{aligned}
& \omega_{m^{\prime} m}=\frac{E_{m^{\prime}}-E_{m}}{\hbar} \\
& =\left(m^{\prime}-m\right) \cdot\left[\left(-\gamma B_{0}+a_{D}\right)+\left(m^{\prime}+m\right) a_{Q}\right]
\end{aligned}
$$

and

$$
\begin{aligned}
& \bar{g}\left(\Omega-\omega_{m^{\prime} m}\right)=\frac{1}{\pi i} \int_{0}^{\infty} d t \int_{-\infty}^{\infty} d a_{D} \\
& \cdot \int_{-\infty}^{\infty} d a_{Q} e^{-i\left(\Omega-\omega_{\left.m^{\prime} m\right) t}\right.} g_{D}\left(a_{D}\right) g_{Q}\left(a_{Q}\right)
\end{aligned}
$$

is the normalized $c$-number line-shape function with

$$
g=g^{\prime}-i g^{\prime \prime} \quad \text { and } \quad \int_{-\infty}^{\infty} d \Omega g(\Omega)=-i .
$$

In deriving (20) we have substituted $N_{m}, N_{m^{\prime}}$ by the unperturbed values $N_{m}^{(0)}, N_{m^{\prime}}^{(0)}$, since in our model the contributions of $a_{D}$ and $a_{Q}$ to the occupation numbers are negligible small.

\section{NAR Absorption and NAR Dispersion}

In order to derive explicit expressions for the ultrasonic attenuation shift caused by a perturbation Hamiltonian of the form (1), it is usually assumed that the resulting change in the phonon loss rate $1 / \tau$ is proportional to the time averaged value of the power absorbed by the nuclei themselves. However, as has been pointed out by Miller et al. [10], this assumption in general leads to results which are not in agreement with experiment so that in the theoretical work dealing with ultrasonic attenuation and sound velocity, some more care should be bestrowed upon the question of physical and experimental reality. In fact, as only those changes in the acoustic energy can be detected at the transducer which result in a synchronous change of the electric impedance $Z$ of the composite resonator (formed by the sample with transducer), phase effects must be considered which usually are neglected when specializing the analysis to time averaged quantities only.

In a previous paper [24] we have shown that in NAR the real part of the fractional change $\Delta_{N} Z / Z_{0}$ gives the NAR absorption and the imaginary part of $\Delta_{N} Z / Z_{0}$ the NAR dispersion, where $Z_{0}$ is the electric impedance without nuclear spin transitions. Taking into account that the acoustic power $P(t)$ fed into the sample is given by $P(t)=U(t) I(t)$, where $I(t)$ and $U(t)$, respectively, are the electric current and the electric voltage at the transducer, and regarding that in $c w$ acoustic resonance experiments $I(t)$ is a harmonic function of time (i.e. $I(\Omega)=\hat{I}(\Omega) \cdot(\delta(\Omega-\omega)$ $+\delta(\Omega+\omega)) / 2$ with $\left.\hat{I}(-\Omega)=\hat{I}^{*}(\Omega)\right)$, by aid of the convolution theorem and $U(\Omega)=Z(\Omega) I(\Omega)$ it is easy to show that at $\Omega=\omega$ the electric impedance $Z(\omega)$ may be written as

$$
Z(\omega)=2 P(2 \omega) /(\hat{I}(\omega) I(\omega))
$$

where $P(2 \omega)$ stands for the Fourier transform of $P(t)$ at $\Omega=2 \omega$ which, in the time space, is equivalent to the reversible part of $P(t)$ (oscillating with $2 \omega$ ) since the irreversibly dissipated power terms have a nonvanishing Fourier component at $\Omega=0$ only. Applying (24) to NAR, the resulting fractional change of the electric impedance is given by

$$
\Delta_{N} Z / Z_{0}=P_{N}(2 \omega) / P_{0}(2 \omega)
$$


where $P_{0}$ stands for the acoustic energy transfer per unit time in the absence of nuclear spin transitions and $P_{N}(2 \omega)$ for $P(2 \omega)-P_{0}(2 \omega)$ which - in the angular frequency-space - is equivalent to the Fourier transform (at $\Omega=2 \omega$ ) of the power $P_{N}(t)=\operatorname{Tr}\{\rho \partial \mathrm{h} / \partial t\}$ absorbed by the nuclear spin system. Combining this expression with Eqs. (9) and (13) and bearing in mind that in NAR the nonzero component $\varepsilon^{\prime}(\vec{r}, t)$ of the not symmetrized strain tensor is of the form $\varepsilon^{\prime}(\vec{r}, t)$ $=\hat{\varepsilon}^{\prime}(\vec{r}) f_{\varepsilon}(t)$ (where $f_{\varepsilon}(t)$ is a dimensionless harmonic function of time with $f_{\varepsilon}(\Omega)=\hat{f}_{\varepsilon}(\Omega)\{\delta(\Omega-\omega)+$ $\delta(\Omega+\omega)\} / 2$ and $\left.\hat{f}_{\varepsilon}(-\Omega)=\hat{f}_{\varepsilon}^{*}(\Omega)\right)$, we obtain

$P_{N}(2 \omega)=-\frac{\rho_{s} c_{a}^{2}}{2} i \omega \chi_{\mathrm{NAR}}(\omega) \hat{f}_{\varepsilon}(\omega) f_{\varepsilon}(\omega) \int_{\left(V_{s}\right)} d^{3} \vec{r}\left[\hat{\varepsilon}^{\prime}(\vec{r})\right]^{2}$.

Inserting (24) and (26) into (25) and using for $I(\Omega)$ and $f_{\varepsilon}(\Omega)$ the expressions given above, the relative change of the electric impedance becomes

$\frac{\Delta_{N} Z(\omega)}{Z_{0}(\omega)}=\frac{\rho_{s} c_{a}^{2} \int_{\left(V_{s}\right)} d^{3} \vec{r}\left(\hat{\varepsilon}^{\prime}(\vec{r})\right)^{2}}{Z_{0}(\omega)}\left|\frac{\hat{f}_{\varepsilon}(\omega)}{\hat{I}(\omega)}\right|^{2} i \omega \chi_{\mathrm{NAR}}(\omega)$

where we have made use of the fact that in NAR the ratio $\hat{f}_{\varepsilon}(\Omega) / \hat{I}(\Omega)$ must be an imaginary quantity since for piezoelectric transducers $I(t)$ is found to be proportional to $d f_{\varepsilon}(t) / d t$.

Since in NAR the experiments are performed at an acoustic standing wave resonance frequency $\omega_{n} / 2 \pi$, we specialize our further considerations to the vicinity of $\omega_{n}$ where $Z_{0}$ is a real quantity and the time averaged value $\bar{P}$ of the electric power is

$\bar{P}=|\hat{I}(\omega)|^{2} Z_{0} / 2=E_{a} / \tau$.

Here $1 / \tau$ is the phonon loss rate and

$E_{a}=E_{a}(V)=\int_{(V)} d^{3} \vec{r} \rho(\vec{r}) c_{a}^{2}(\vec{r}) \cdot\left|\hat{\varepsilon}^{\prime}(\vec{r}) \hat{f}_{\varepsilon}(\omega)\right|^{2} / 2$

is the total acoustic energy stored in the composite resonator whose volume $V$ (including both the sample and the transducer) is larger than $V_{s}$. Combining these expressions with (27), for $\omega \approx \omega_{n}$ the result is

$\frac{\Delta_{N} Z(\omega)}{Z_{0}(\omega)}=i \eta_{a} Q_{a} \cdot \chi_{\mathrm{NAR}}(\omega)$

where $Q_{a}=\omega_{n} \tau$ is the acoustic quality factor, $\eta_{a}$ $=E_{a}\left(V_{s}\right) / E_{a}(V)$ is the acoustic filling factor and $E_{a}\left(V_{s}\right)$ is the acoustic energy stored in the sample containing the nuclei. Consequently, the filling factor is of the order 1 if the mass of the transducer plus bond is small against the mass of the ultrasonically excited specimen. On the other hand, from the equation of motion of an infinitesimal element of volume it is easy to show [25] that in the vicinity of $\omega_{n}$ the fractional change in the electric impedance is

$\frac{\Delta_{N} Z}{Z_{0}}=\frac{Q_{a}}{\omega}\left[\Delta_{N}(1 / \tau)-2 i\left(\Delta_{N} \omega_{n}\right)\right]$.

Hence, combining (28) and (29) and using the definition

$\chi_{\mathrm{NAR}}=\chi_{\mathrm{NAR}}^{\prime}-i \chi_{\mathrm{NAR}}^{\prime \prime}$

with $\Delta \omega_{n} / \omega_{n}=\Delta c_{a} / c_{a}$ the actual shift (observed in experiment) of both, the phonon loss rate $1 / \tau$ (NAR absorption) and acoustic phase velocity $c_{a}$ (NAR dispersion), comes out to be

$\Delta_{N}(1 / \tau)=\eta_{a} \omega \chi_{\mathrm{NAR}}^{\prime \prime} \quad \Delta_{N} c_{a}=-\frac{\eta_{a} c_{a}}{2} \chi_{\mathrm{NAR}}^{\prime}$

thus relating microscopic to macroscopic quantities which are relevant in NAR.

\section{Results and Discussion}

Given the ultrasonic attenuation and velocity shift in terms of $\chi_{\mathrm{NAR}}^{\prime}$ and $\chi_{\mathrm{NAR}}^{\prime \prime}$, we now present explicit expressions for the different NAR susceptibilities defined in part 2 .

\subsection{NAR-Dipole Susceptibility}

Starting from the defining Eq. (14a), by aid of Parseval's identity and with the help of the familiar nuclear magnetic spin susceptibility $\chi=\chi^{\prime}-i \chi^{\prime \prime}$ which may be defined as

$\chi(\Omega)=-\left(\frac{1}{i \hbar}\right) \frac{\operatorname{Tr}\left\{\rho_{0}\left[\mathrm{Ih}_{D}(\Omega), \tilde{\mathrm{Ih}}_{D}(\Omega)\right]\right\}}{\int_{\left(V_{s}\right)} d^{3} \vec{r}\left[b_{\perp}(\vec{r}, \Omega)\right]^{2} / \mu_{0}}$

the NAR-dipole susceptibility can be brought into the form

$\chi_{\mathrm{NAR}}^{(D)}(\Omega)=\frac{\int d^{3} \vec{k} b_{\perp}(\vec{k}, \Omega) b_{\perp}(-\vec{k}, \Omega)}{\rho_{s} c_{a}^{2} \mu_{0} \int d^{3} \vec{k} \varepsilon^{\prime}(\vec{k}, \Omega) \varepsilon^{\prime}(-\vec{k}, \Omega)} \chi(\Omega)$

where $b_{\perp}$ is the projection of the sound induced $\mathrm{rf}$ magnetic field $\vec{b}$ on a plane perpendicular to $\vec{B}_{0}$ and the argument $\vec{k}$ stands for the space-Fourier-transform. Here, only the component $b_{\perp}$ appears because the parallel part is of no interest as an NMR coupling. Since in (33) $b_{\perp}(\vec{k}, \Omega)$ and $\varepsilon^{\prime}(\vec{k}, \Omega)$ are $c$-number quantities, it is evident that any phase shift of $\int d^{3} \vec{k} b_{\perp}(\vec{k}, \Omega) b_{\perp}(-\vec{k}, \Omega)$ with respect to $\int d^{3} \vec{k} \varepsilon^{\prime}(\vec{k}, \Omega) \varepsilon^{\prime}(-\vec{k}, \Omega)$ will lead to asymmetric NAR absorption (and NAR dispersion) signals since both 
$\chi^{\prime}$ and $\chi^{\prime \prime}$ will contribute to $\chi_{\mathrm{NAR}}^{\prime \prime},\left(\chi_{\mathrm{NAR}}^{\prime}\right)$. Although Eq. (33) may be applied to solid as well as to liquid metals [26], for a more detailed analysis a relation between $b_{\perp}$ and $\varepsilon^{\prime}$ (or $\vec{v}_{i}$ ) must be known which may be derived [27] from Maxwell's equations by a straight forward calculation using the usual definition of electronic current and the Boltzmann equation in the relaxation time approximation. On the other hand, as in conventional NAR experiments the ultrasonic frequencies $\omega / 2 \pi$ are in the megacycle range and $\omega_{c} \tau, l / \lambda$ and $\omega \tau$ usually are negligible small against 1 , we may restrict our further considerations to the "Alpher-Rubin" (AR) limit [28] where, as a consequence of the Landau orbital term, the sound induced rf magnetic field component becomes [16]*

$b_{\perp}^{(\mathrm{AR})}(\vec{k}, \Omega)=\frac{B_{0}}{1-i \beta(\Omega)} \cos \theta \sin \Phi \varepsilon^{\prime}(\vec{k}, \Omega)$.

Here $\omega_{c}$ is the cyclotron angular frequency, $l$ the electron mean free path, $\lambda$ the ultrasonic wave length, $\beta=\Omega /\left(\mu_{0} \sigma_{0} c_{a}^{2}\right), \sigma_{0}$ the $d c$ conductivity, $\theta$ is the angle between the acoustic wave vector and $\vec{B}_{0}$, and $\Phi$ the angle between the lattice displacement and $\vec{B}_{0}$. On the other hand, as has been pointed out by Niez and Averbuch [15], there is still another magnetic field term $\vec{b}^{(P)}$ whose source is the variation with strain of the Van Vleck [29] orbital paramagnetism or the spin-orbit coupling. Using an isotropic model for cubic crystals and keeping only parts coming from shear strains, the result is [15]

$\vec{b}^{(P)}(\vec{k}, \Omega)=i K_{a}\left\{\left(\vec{u}_{i}(\vec{k}, \Omega) \cdot \vec{B}_{0}\right) \vec{k}+\left(\vec{k} \cdot \vec{B}_{0}\right) \vec{u}_{i}(\vec{k}, \Omega)\right\}$

where $\vec{u}_{i}$ is the lattice displacement and $K_{a}$ is the coupling coefficient relating the transverse Knight shift to shear strains. However, with the exception of those metals (like $\mathrm{Pt}$ ) where both the paramagnetostrictive effect [30] and the Knight shift [31] are large, the contribution of $\vec{b}^{(P)}$ to the sound induced $\mathrm{rf}$ magnetic field is negligible small. Hence, combining (33) and (34), the calculation yields

$\chi_{\mathrm{NAR}}^{(D)}=\frac{B_{0}^{2} \cos ^{2} \theta \sin ^{2} \Phi}{\mu_{0} \rho_{s} c_{a}^{2}}\left(\frac{1}{1+\beta^{2}}\right)^{2}\left\{\left(1-\beta^{2}\right)+2 i \beta\right\} \chi$

which, in the limits where band structure effects are negligible small, agrees well with experiment [32] and with the final expressions of actual classical treatments $[11,13,16]$ of dipole-NAR in metals $\star \star$.

* It is interesting to note that the physical origin of the AR-field is the lattice displacement $\vec{u}_{i}$ and not the elastic strain $\overleftrightarrow{\varepsilon}$. However, as in the $\Omega-\vec{k}$-Fourier space the identity $\left.\overleftrightarrow{\varepsilon}^{\prime}=i \vec{k}\right)\left(\vec{u}_{i}\right.$ must hold, $b_{\perp}^{(\mathrm{AR})}$ may be expressed by the nonzero strain component $\varepsilon^{\prime}$

$\star \star$ The reader who is interested in a detailed analysis of (36) is referred to Refs. 13 and 16
We may emphasize that the validity of (33) is not restricted to the AR limit so that band structure effects modifying $\vec{b}(\vec{r}, t)$, can easily be incorporated in $\chi_{\text {NAR }}^{(D)}(\Omega)$ [33]. Furthermore, it is worth mentioning that the theory presented here, to our knowledge gives the first quantum theoretical treatment of dipole-NAR in conductors whose results are in agreement with experiment. Finally, we would like to point out that the sound induced rf magnetic field is an intrinsic field so that dipole-NAR may be regarded as NMR in the interior of a metal. Hence, dipole-NAR has the distinct advantage over NMR in that the difficulties resulting from skin effect are avoided.

\subsection{NAR-Quadrupole Susceptibility}

Choosing a coordinate system $(x, y, z)$ having $z$ along $\vec{B}_{0}$ and using the abbreviations

$\mathbb{Q}_{0}=\alpha_{Q}\left\{3 \Pi_{z}^{2}-I(I+1)\right\} \quad V_{0}(\vec{r}, t)=V_{z z}$ $\mathbb{Q}_{ \pm 1}=\alpha_{Q}\left\{\mathbb{I}_{z} \mathbb{I}_{ \pm}+\mathbb{I}_{ \pm} \mathbb{I}_{z}\right\} \quad V_{ \pm 1}(\vec{r}, t)=V_{x z} \pm i V_{y z}$ $\mathbb{Q}_{ \pm 2}=\alpha_{Q} \mathbb{I}_{ \pm}^{2} \quad V_{ \pm 2}(\vec{r}, t)=\frac{1}{2}\left\{\left(V_{x x}-V_{y y}\right) \pm 2 i V_{x y}\right\}$

where $\alpha_{Q}=e_{0} Q /(4 I(2 I-1))$, the corresponding expression for the quadrupolar perturbation Hamiltonian $\hat{\mathbb{h}}_{Q}$ of a single spin may be written as [23]

$\widehat{\mathrm{h}}_{Q}(\Omega)=\sum_{\lambda=-2}^{2} \mathbb{Q}_{\lambda} V_{-\lambda}(\vec{r}, \Omega)$.

Hence, combining (3), (14b), (20), (37) and (38) we obtain

$\chi_{\text {NAR }}^{(Q)}(\Omega)=\frac{\pi}{\hbar \rho_{s} c_{a}^{2}} \sum_{\lambda=-2}^{2} \frac{\int_{\left(V_{s}\right)} d^{3} \vec{r} V_{\lambda}(\vec{r}, \Omega) V_{-\lambda}(\vec{r}, \Omega)}{\int_{\left(V_{s}\right)} d^{3} \vec{r}\left[\varepsilon^{\prime}(\vec{r}, \Omega)\right]^{2}}$
$\cdot \sum_{m=-I}^{I} \frac{N_{m}^{(0)}-N_{m+\lambda}^{(0)}}{V_{s}} \cdot\left|\left\langle m+\lambda\left|\mathbb{Q}_{\lambda}\right| m\right\rangle\right|^{2} g\left(\Omega-\omega_{m+\lambda, m}\right)$

where we have made use of the identities

$\mathbb{I}_{ \pm}|m\rangle=f_{I}( \pm m)|m \pm 1\rangle$,

$\mathbb{I}_{ \pm}^{2}|m\rangle=f_{I}( \pm m) f_{I}(1 \pm m)|m \pm 2\rangle$,

with

$f_{I}(m)=\{I(I+1)-m(1+m)\}^{1 / 2}$

and

$\left\langle m^{\prime}\left|\mathbb{Q}_{\lambda}\right| m\right\rangle\left\langle m\left|\mathbb{Q}_{\lambda^{\prime}}\right| m^{\prime}\right\rangle=\delta_{\lambda^{\prime},-\lambda} \delta_{m^{\prime}, m+\lambda}$ $\cdot\left|\left\langle m^{\prime}\left|\mathbb{Q}_{\lambda}\right| m\right\rangle\right|^{2}$.

Hence, from (37) to (41) it becomes obvious that both nuclear acoustic spin transitions $|\Delta m|=1$ and $|\Delta m|=2$ 
may be excited in quadrupole-NAR, so that $\chi_{\mathrm{NAR}}^{(Q)}$ must be of the form

$$
\chi_{\mathrm{NAR}}^{(Q)}=\chi_{\mathrm{NAR}}^{(Q 1)}+\chi_{\mathrm{NAR}}^{(Q 2)} .
$$

Here the indices 1, 2 stand, respectively, for nuclearelectric-quadrupole transitions $|\Delta m|=1$ and $|\Delta m|=2$, where

$\chi_{\text {NAR }}^{(Q 1)}(\Omega)=D_{1}(\Omega) \frac{\int_{\left(V_{s}\right)} d^{3} \vec{r} V_{1}(\vec{r}, \Omega) V_{-1}(\vec{r}, \Omega)}{\int_{\left(V_{s}\right)} d^{3} \vec{r}\left[\varepsilon^{\prime}(\vec{r}, \Omega)\right]^{2}}$

with

$$
\begin{aligned}
& D_{1}(\Omega)=\frac{\pi \alpha_{Q}^{2}}{\hbar \rho_{s} c_{a}^{2}} \sum_{m=-I}^{I-1} \frac{N_{m}^{(0)}-N_{m+1}^{(0)}}{V_{s}} \cdot(2 m+1)^{2} f_{I}^{2}(m) \\
& \cdot\left\{g\left(\Omega-\omega_{m+1, m}\right)-g\left(\Omega+\omega_{m+1, m}\right)\right\}
\end{aligned}
$$

and

$\chi_{\mathrm{NAR}}^{(Q 2)}(\Omega)=D_{2}(\Omega) \frac{\int_{\left(V_{s}\right)} d^{3} \vec{r} V_{2}(\vec{r}, \Omega) V_{-2}(\vec{r}, \Omega)}{\int d^{3} \vec{r}\left[\varepsilon^{\prime}(\vec{r}, \Omega)\right]^{2}}$

with

$$
\begin{aligned}
& D_{2}(\Omega)=\frac{\pi \alpha_{Q}^{2}}{\hbar \rho_{s} c_{a}^{2}} \cdot \sum_{m=-I}^{I-2} \frac{N_{m}^{(0)}-N_{m+2}^{(0)}}{V_{s}} f_{I}^{2}(m) f_{I}^{2}(1+m) \\
& \cdot\left\{g\left(\Omega-\omega_{m+2, m}\right)-g\left(\Omega+\omega_{m+2, m}\right)\right\} .
\end{aligned}
$$

Since the DEFG tensor components (and consequently the DEFG terms $V_{0}, V_{ \pm 1}$ and $V_{ \pm 2}$ ) are linear response quantities with respect to the elastic strain tensor, from (43) and (44) it becomes evident that the experimental determination of $\chi_{\mathrm{NAR}}^{(Q 1)}$ and $\chi_{\mathrm{NAR}}^{(Q 2)}$ does not require the knowledge of the elastic strain amplitude. The relation between the DEFG and the symmetrized elastic strain tensor $\overleftrightarrow{\varepsilon}=\left\{\overleftrightarrow{\varepsilon^{\prime}}+\left(\overleftrightarrow{\varepsilon}^{\prime}\right)^{+}\right\} / 2$ (where $\left({\overleftrightarrow{\varepsilon^{\prime}}}^{\prime}\right)^{+}$is the adjoint of $\overleftrightarrow{\varepsilon}^{\prime}$ ) is represented by the fourth-rank tensor $\overleftrightarrow{S}$ [34] yielding

$$
V_{i j}=\sum_{k, l} S_{i j k l} \varepsilon_{k l}
$$

Hence, as $\overleftrightarrow{\varepsilon}$ is an external quantity, there is no loss of information if either the $S$ tensor or the dynamic electric field gradient tensor $\overleftrightarrow{V}$ is determined. In the long-wave limit $S_{i j k l}$ are real quantities and are independend of $\vec{k}$ and $\Omega$ [35]. For cubic crystals and in a coordinate system $(X, Y, Z)$ having its axes along the cubic unit cell, Eq. (45) gets the much simpler form [34]

$$
\begin{aligned}
& V_{X X}=S_{11} \varepsilon_{X X}+S_{12}\left(\varepsilon_{Y Y}+\varepsilon_{Z Z}\right) \quad \text { cyclic } \\
& V_{X Y}=S_{44}\left(\varepsilon_{X Y}+\varepsilon_{Y X}\right)
\end{aligned}
$$

where $S_{11}, S_{12}$ and $S_{44}$ are the three distinct components of the fourth-rank tensor $\overleftrightarrow{S}$ in Voigt notation.
However, the number of independent components required to describe the quadrupole coupling between the elastic strains and the nuclear spin system is only two [36], of which one is $S_{44}$ and the other $S_{11}-S_{12}$. We shall not give explicitly the various transformations [37] necessary in order to transform (46) into the $(x, y, z)$-magnet system but simply present the results for cases of wide practical relevance where longitudinal acoustic waves are propagated along the [110] crystal axis and the applied magnetic field $\vec{B}_{0}$ is rotated in different crystal planes. If $\varphi$ is the angle between $\vec{B}_{0}$ and the [001] axis and $\theta$ the angle between the acoustic wave vector and $\vec{B}_{0}$, by combining (37), (43) and (44) the following cases may be distinguished.

\section{a) $\vec{B}_{0}$ Rotated in the (110)-Plane}

$$
\begin{aligned}
& \chi_{\mathrm{NAR}}^{(Q 1)}(\Omega)=\frac{1}{4} \cdot D_{1}(\Omega)\left[\left(S_{11}-S_{12}\right)-2 S_{44}\right]^{2} \sin ^{2} \varphi \cos ^{2} \varphi \\
& \chi_{\mathrm{NAR}}^{(Q 2)}(\Omega)=\frac{1}{16} \cdot D_{2}(\Omega)\left[\left(S_{11}-S_{12}\right) \cdot \sin ^{2} \varphi\right. \\
& \left.+2 S_{44}\left(1+\cos ^{2} \varphi\right)\right]^{2}
\end{aligned}
$$

b) $\vec{B}_{0}$ Rotated in the (110)-Plane

$\chi_{\mathrm{NAR}}^{(Q 1)}(\Omega)=\frac{1}{4} \cdot D_{1}(\Omega)\left[\left(S_{11}-S_{12}\right)+2 S_{44}\right]^{2} \sin ^{2} \theta \cos ^{2} \theta$

$\chi_{\mathrm{NAR}}^{(Q 2)}(\Omega)=\frac{1}{16} \cdot D_{2}(\Omega)\left[\left(S_{11}-S_{12}\right) \cdot \cos ^{2} \theta\right.$

$\left.-2 S_{44}\left(1+\sin ^{2} \theta\right)\right]^{2}$

c) $\vec{B}_{0}$ Rotated in the (001)-Plane

$\chi_{\mathrm{NAR}}^{(Q 1)}(\Omega)=\frac{1}{4} \cdot D_{1}(\Omega)\left[4 S_{44}\right]^{2} \sin ^{2} \theta \cos ^{2} \theta$

$\chi_{\mathrm{NAR}}^{(Q 2)}(\Omega)=\frac{1}{16} \cdot D_{2}(\Omega)\left[\left(S_{11}-S_{12}\right)+2 S_{44}\left(1-2 \cos ^{2} \theta\right)\right]^{2}$.

Hence, from absolute measurements of the absorptive or dispersive parts of either $\chi_{\mathrm{NAR}}^{(Q 1)}$ or $\chi_{\mathrm{NAR}}^{(Q 2)}$ it is possible to determine the relevant $S$ tensor components. It should be noticed, however, that the NAR-quadrupole susceptibilities are bilinear in the relevant $S$ tensor components so that, besides the absolute values, only the relative sign of $S_{11}-S_{12}$ and $S_{44}$ can be determined from $\chi_{\mathrm{NAR}}^{(Q)}$.

\subsection{NAR-Interference Term}

Starting from the defining equation (14c) and using Parseval's identity, with equations (20), (37) to (41), 
$\widehat{\mathrm{h}}_{D}=-\gamma \hbar\left\{b_{x}\left(\mathrm{II}_{+}+\mathrm{II}_{-}\right)-i b_{y}\left(\mathrm{II}_{+}-\mathrm{II}_{-}\right)\right\} / 2$ and the identity $f_{I}( \pm m)=f_{I}(-1 \mp m)$, a tedious but straight forward calculation then yields

$$
\begin{aligned}
& \chi_{\mathrm{NAR}}^{(\mathrm{Int})}(\Omega)=D_{11}(\Omega) \\
& \cdot \frac{\int d^{3} \vec{k}\left\{b_{x}(\vec{k}, \Omega) V_{x z}(-\vec{k}, \Omega)+b_{y}(\vec{k}, \Omega) V_{y z}(-\vec{k}, \Omega)\right\}}{\int d^{3} \vec{k} \varepsilon^{\prime}(\vec{k}, \Omega) \varepsilon^{\prime}(-\vec{k}, \Omega)}
\end{aligned}
$$

with

$D_{11}(\Omega)=-\frac{\pi \gamma \alpha_{Q}}{\rho_{s} c_{a}^{2}} \sum_{m=-I}^{I-1} \frac{N_{m}^{(0)}-N_{m+1}^{(0)}}{V_{s}} \cdot(2 m+1) f_{I}^{2}(m)$

$\cdot\left\{g\left(\Omega-\omega_{m+1, m}\right)-g\left(\Omega+\omega_{m+1, m}\right)\right]$.

Consequently the interference term does not influence the $|\Delta m|=2$ NAR signals, but contributes to the $|\Delta m|=1$ NAR lines only. Furthermore $\chi_{\mathrm{NAR}}^{(\mathrm{Int})}$ will vanish if either $\chi_{\mathrm{NAR}}^{(D)}$ or $\chi_{\mathrm{NAR}}^{(Q 1)}$ are zero. Confining ourselves to the same orientation as in the previous section and regarding that $b_{\perp}=0$ for $\theta=\pi / 2$, with (34), (46), (50) and $\theta$ defined as above, for longitudinal sound propagated along the [110] axis we obtain

a) $\vec{B}_{0}$ Rotated in the (110)-Plane

$\chi_{\mathrm{NAR}}^{(\mathrm{Int})}(\Omega)=0$

b) $\vec{B}_{0}$ Rotated in the (110)-Plane

$$
\begin{aligned}
& \chi_{\mathrm{NAR}}^{\text {(Int) }}(\Omega)=\frac{D_{11}(\Omega)}{2} \\
& \cdot \frac{B_{0}\left\{\left(S_{11}-S_{12}\right)+2 S_{44}\right\} \sin ^{2} \theta \cos ^{2} \theta}{(1-i \beta)}
\end{aligned}
$$

c) $\vec{B}_{0}$ Rotated in the (001)-Plane

$$
\chi_{\mathrm{NAR}}^{(\mathrm{Int})}(\Omega)=D_{11}(\Omega) \cdot \frac{2 B_{0} S_{44} \sin ^{2} \theta \cos ^{2} \theta}{(1-i \beta)} .
$$

The most striking feature of these expressions is that they are linear in $\gamma \cdot Q$ and in the relevant $S$ tensor components, so that $\chi_{\mathrm{NAR}}^{(\mathrm{Int})}$ reverses sign if either the sign of the $S$ tensor components or the relative sign of $\gamma$ and $Q$ is altered. Hence, provided that the relative sign of $\gamma$ and $Q$ is known, NAR investigations of the interference term should reveal the sign of both $\left(S_{11}-S_{12}\right)$ and $S_{44}$. Furthermore it is interesting to note that in contrast to $\chi_{\mathrm{NAR}}^{(D)}$ and $\chi_{\mathrm{NAR}}^{(Q)}$, nuclear spin transitions of the same kind - like $\{m \rightarrow m+1\}$ and $\{-(m+1) \rightarrow-m\}-$ now do contribute to $\chi_{\mathrm{NAR}}^{(\text {Int) }}$ with different signs (see $\left.(50)\right)$ because $(2 m$ $+1)$ reverses sign if $m$ is substituted by $-(m+1)$ whereas $f_{I}(m)$ and $N_{m}^{(0)}-N_{m+1}^{(0)}$ remain unchanged.

To become more familiar with the interference term we give the final results if $g_{D}\left(a_{D}\right)$ and $g_{Q}\left(a_{Q}\right)$ (see (16) and (22)) are Gaussian distribution functions with
$g_{Q}\left(a_{Q}\right)$ centered around $a_{Q}^{(0)}>0$. For $\beta=0$ (i.e. for low temperatures and very pure metals), $I=\frac{7}{2}$ and $\Omega, \gamma$, $Q>0$, the calculation then yields the variation with $B_{0}$ of the absorptive parts of $\chi_{\mathrm{NAR}}^{(D)}, \chi_{\mathrm{NAR}}^{(Q 1)}$ and $\chi_{\mathrm{NAR}}^{(\mathrm{Int})}$ as shown in Fig. 1.

From Fig. 1 it is evident that the appearance of the interference term results in an asymmetric line-shape of the absorptive part $\left(\chi_{\mathrm{NAR}}^{\prime \prime}\right)$ of the total $|\Delta m|$ $=1 \mathrm{NAR}$ signal which will pass into its mirror-image if either the relative sign of $\gamma$ and $Q$ or the sign of the contributing $S$ tensor components is reversed. Furthermore it becomes obvious that in perfect cubic crystals, where $a_{Q}=0$ and the single lines (see (21), (22)) are no longer separated or distinguished by their line-shapes, the interference term will vanish. In real crystals, however, there exists a distribution of $a_{Q}$ due
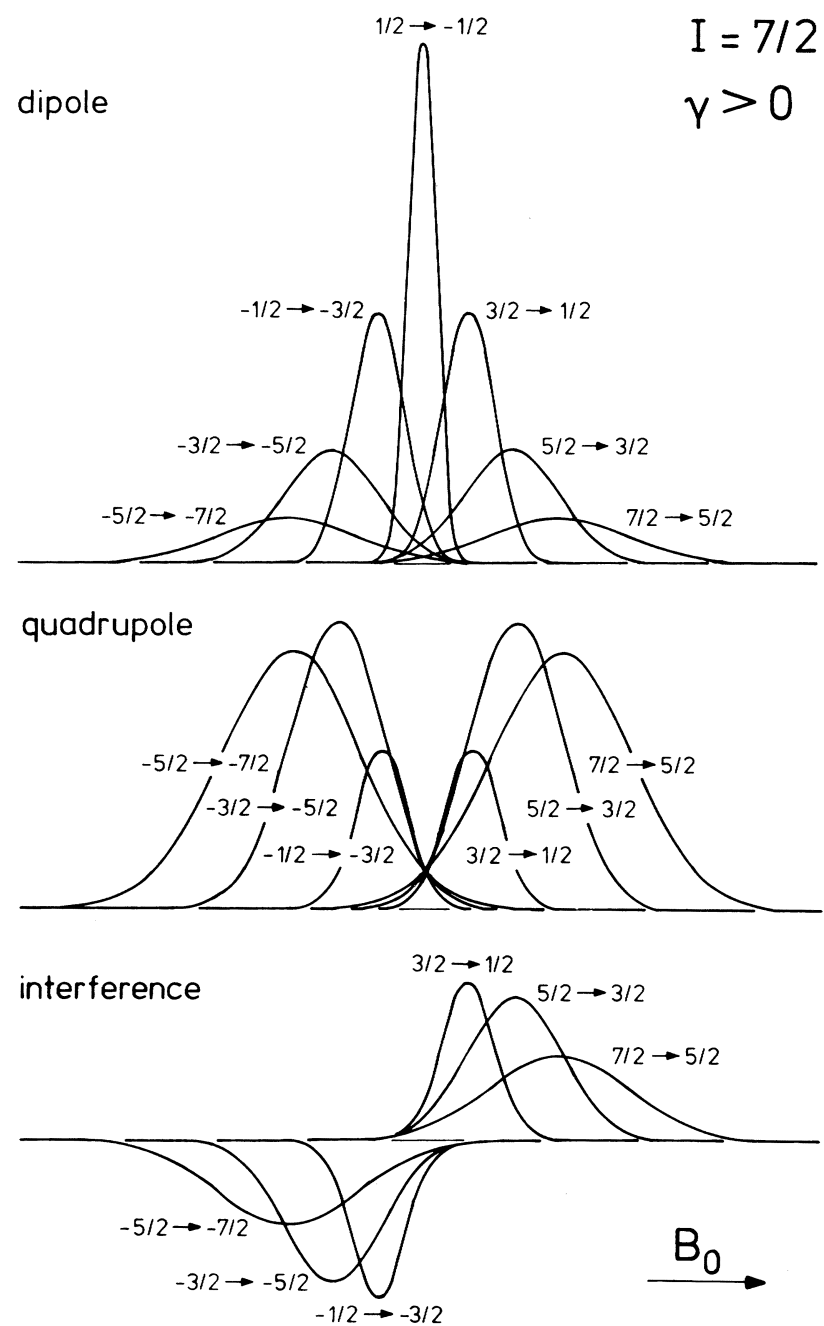

Fig. 1. Pattern of the various contributions to the $|\Delta m|=1$ NAR absorption signal for $a_{Q}^{(0)}>0$. The full half line widths of the inhomogeneously broadened single lines $\left(m \rightarrow m^{\prime}\right)$ are $\left[\Delta_{D}^{2}+\Delta_{Q}^{2}(2 m\right.$ $\left.-1)^{2}\right]^{1 / 2}$, where $\Delta_{D}$ and $\Delta_{Q}$ are the full half width's of the Gaussian distribution functions $g_{D}\left(a_{D}\right)$ and $g_{Q}\left(a_{Q}\right)$, respectively. Note, that the interference graph is not true to scale 


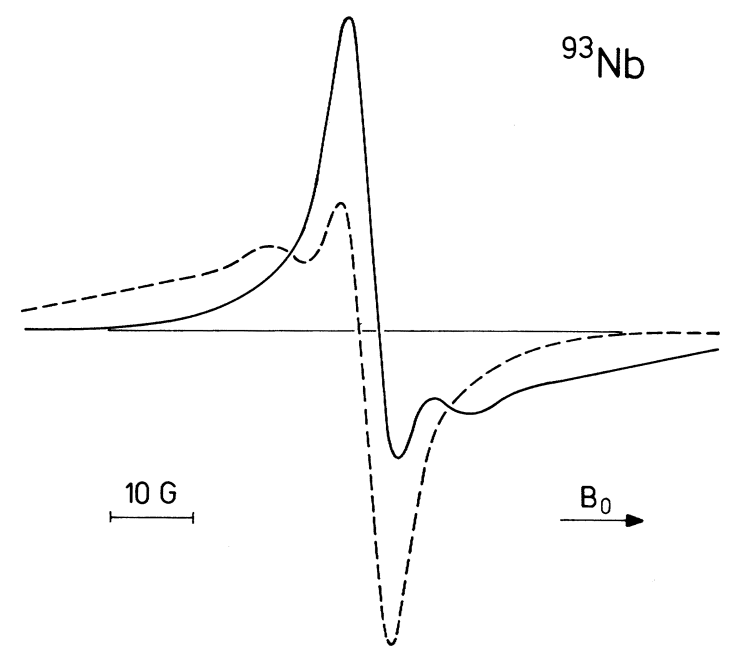

Fig. 2. Derivative of the expected $|\Delta m|=1$ NAR absorption signal of niobium $(I=9 / 2, \gamma \cdot Q<0)$ at low temperatures $(\beta=0)$. The NAR line-shape is calculated from (36), (49a) and (51c) utilizing for $g_{D}\left(a_{D}\right)$ and $g_{Q}\left(a_{Q}\right)$ Gaussian distribution functions with $\left\{\Delta_{D}, a_{D}^{(0)}\right\}$ $=\{7.1,5.0\} \mathrm{kHz}$ and $\left\{\Delta_{Q}, a_{Q}^{(0)}\right\}=\{5.9,2.5\} \mathrm{kHz}$. The full line gives the result for $S_{44}>0$ and the dashed line for $S_{44}<0$

to the presence of lattice imperfections or static strains resulting from external stresses during sample preparation, so that in real cubic metals interference effects in general cannot be neglected. In order to demonstrate the influence of the interference term on the expected NAR lines $\left(\propto \partial \chi_{\mathrm{NAR}}^{\prime \prime} / \partial B_{0}\right)$ in metals, we have calculated $\partial \chi_{\mathrm{NAR}}^{\prime \prime} / \partial B_{0}$ of ${ }^{93} \mathrm{Nb}$ in a real (statically strained) [110]-single crystal of niobium for both $S_{44}>0$ and $S_{44}<0$. The distribution functions $g_{D}\left(a_{D}\right)$ and $g_{Q}\left(a_{Q}\right)$ were assumed to be Gaussian and their widths and center-frequencies were determined in our laboratory. For longitudinal acoustic waves and $\vec{B}_{0} \perp[001]$ the results are shown in Fig. 2 .

Since there is a marked difference in the calculated NAR lines for either $S_{44}>0$ or $S_{44}<0$, from Fig. 2 and the statements given in Sect. 4.2 it is evident that there should be no difficulties to determine both the absolute values and the signs of the relevant $S$ tensor components in $\mathrm{Nb}$. Such experiments, combined with a more detailed analysis of the interference term, are in progress and will be published elsewhere.

We are much indebted to Prof. Dr. S. Wilking for many stimulating discussions. This work was supported by the Deutsche Forschungsgemeinschaft (SFB 161).

\section{References}

1. Bolef, D.I., Menes, M.: Phys. Rev. 114, 1441 (1959)

2. Gregory, E.H., Bömmel, H.E.: Phys. Rev. Lett. 15, 404 (1965)

3. Bolef, D.I. In: Physical Acoustics, Mason, W.P. (Ed.), p. 113, Vol.IVA. New York: Academic Press 1966 (see references therein)

4. Kessel, A.R.: Akustische Kernresonanz. Berlin: Akademie Verlag $\mathrm{GmbH}, 1973$
5. Quinn, J.J., Ying, S.C.: Phys. Lett. 23, 61 (1966)

6. Buttet, J., Gregory, E.H., Baily, P.K.: Phys. Rev. Lett. 23, 1030 (1969)

7. Khenner, E.K.: Sov. Phys. JETP 36, 137 (1973)

8. For isolators of cubic symmetry containing paramagnetic impurities with effective spin $S=1 / 2$ and subjected to the simultaneous action of both a sound wave and an external rf magnetic field of identical frequencies, interference effects of non quadrupolar origin have been treated by:

a) Nagibarov, V.R., Samartsev, V.V., Solovarov, N.K.: Sov. Phys. JETP 34, 873 (1972)

b) Nagibarov, V.R., Samartsev, V.V., Solovarov, N.K.: Sov. Phys. Solid State 13, 2590 (1972)

c) Nagibarov, V.R., Solovarov, N.K., Samartsev, V.V.: Phys. Lett. A 37, 455 (1971)

9. Quinn, J.J.: J. Phys. Chem. Solids 32, 1701 (1970)

10. a) Smith, W.D., Miller, J.G.: Phys. Lett. A33, 389 (1970) b) Miller, J.G., Smith, W.D., Bolef, D.I., Sundfors, R.K.: Phys. Rev. B3, 1547 (1971)

11. Buttet, J.: Solid State Commun. 9, 1129 (1971)

12. Franz, J.R.: Phys. Lett. A37, 197 (1971)

13. Fedders, P.A.: Phys. Rev. B7, 1739 (1973).

Note that there is an error in print in Eq. (20). The correct expression was reported by Leisure, L.L. et al.: Phys. Rev. Lett. 30, 1326 (1969)

14. Buttet, J.: Solid State Commun. 16, 397 (1975)

15. Niez, J.J., Averbuch, P.A.: J. Phys. F5, 1227 (1975)

16. Müller, V., Schanz, G., Fischer, E., Unterhorst, E.J.: Phys. stat. sol. (b) 80, 629 (1977)

17. Müller, V.: Phys. Lett. A 60, 240 (1977)

18. Fischer, E., Müller, V., Ploumbidis, D., Schanz, G.: Phys. Rev. Lett. 40, 796 (1978)

19. Kubo, R., Tomita, K.: J. Phys. Soc. Jap. 9, 888 (1954)

20. Kubo, R.: J. Phys. Soc. Jap. 12, 570 (1957)

21. Kessel, A.R.: thesis, University of Kasan (1962)

22. Butterworth, J.: J. Proc. Phys. Soc. (London) 86, 297 (1965)

23. Kanert, O., Mehring, M. In: NMR, Diehl, P., Fluck, E., Kosefeld, R. (eds.), p. 1, Vol.3. Berlin-Heidelberg-New York: Springer 1971

24. Müller, V.: J. Phys. E8, 127 (1975)

25. Müller, V.: thesis, Freie Universität Berlin, 1973

26. a) Reusche, M.J., Unterhorst, E.J.: Phys. Lett. A 51, 275 (1975) b) Unterhorst, E.J., Müller, V., Schanz, G.: phys. stat. sol. (b) 84, K 53 (1977)

27. Cohen, M.H., Harrison, M.J., Harrison, W.A.: Phys. Rev. 117, $937(1960)$

28. Alpher, R.A., Rubin, R.J.: J. acoust. Soc. America 26, 452 (1954)

29. Van Vleck, J.H.: Phys. Rev. 74, 1168 (1948)

30. Fawcett, E.: Phys. Rev. B2, 3887 (1970)

31. Clogston, A.M., Jaccarino, V., Yafet, Y.: Phys. Rev. 134A, 650 (1964)

32. See references in Ref. 16

33. Müller, V., Unterhorst, E.J., Maurer, D., Schanz, G.: Phys. Lett. A69, 139 (1978)

34. Taylor, E.F., Bloembergen, N.: Phys. Rev. 113, 431 (1959)

35. Cohen, M.H., Reif, R. In: Solid state Physics, Seitz, F., Turnbull, D. (eds.), p. 321, Vol. 5. New York: Academic Press, 1957

36. Harrison, R.J., Sagalyn, P.J.: Phys. Rev. 128, 1630 (1962)

37. Sundfors, R.K.: Phys. Rev. 177, 1221 (1969)

V. Müller

U. Bartell

Institut für Atom- und Festkörperphysik (B)

Freie Universität Berlin

Königin-Luise-Straße 28/30

D-1000 Berlin 33 